\title{
Inflación vectorial en el marco de las teorías de gauge no abelianas
}

\author{
Carlos Nieto ${ }^{1, *}$, Yeinzon Rodríguez ${ }^{1,2}$
}

${ }^{1}$ Escuela de Física, Universidad Industrial de Santander, Ciudad Universitaria, Bucaramanga, Colombia

${ }^{2}$ Centro de Investigaciones en Ciencias Básicas y Aplicadas, Universidad Antonio Nariño, Bogotá, Colombia

\begin{abstract}
Resumen
Se encuentra en la literatura científica que gran parte de los modelos inflacionarios carecen de soportes observacionales o conceptuales. Es así como recientes observaciones sobre una posible dirección privilegiada en el universo implican que no es conveniente tomar campos escalares como generadores de inflación. Por otra parte, los recientes modelos inflacionarios que emplean campos vectoriales requieren modificar la gravedad o las condiciones muy finas para su apropiado comportamiento; además, carecen de naturalidad y no presentan una de las características más importantes de las teorías de la física moderna: la existencia de simetrías internas. En este artículo se presenta un modelo inflacionario vectorial enmarcado en una teoría de gauge $S U(2)$ compuesto por tres campos vectoriales sobre los cuales se hace una suposición bien fundamentada con respecto a su dirección, su norma, y su dependencia temporal. Lo anterior permite obtener una era de inflación isótropa en un fondo rotacionalmente invariante si se tiene en cuenta la relación homomórfica entre los grupos $S U(2)$ y $O(3)$. Se estudia, en este escenario, la dinámica de los campos vectoriales y las condiciones para tener expansión acelerada; además, se analiza la evolución del sistema en el régimen de rodadura lenta y se obtienen soluciones numéricas de las ecuaciones de movimiento. Debido a la naturalidad del modelo, los resultados numéricos observados y la generalidad de la suposición propuesta, se concluye que este tipo de inflación representa de manera adecuada el comportamiento del universo primordial a la luz de las más recientes observaciones.
\end{abstract}

Palabras clave: paradigma inflacionario, campos vectoriales, teorías de gauge no abelianas, inflación del tipo rodadura lenta.

\section{Vector inflation in the framework of non-Abelian gauge theories}

\begin{abstract}
According to the literature, there is a significant number of inflationary models that lack observational and conceptual support. Such is the case with recent observations regarding a possible preferred direction in the universe, which imply that scalar fields are not suitable as generators of inflation. On the other hand, recent inflationary models that employ vector fields require a modification of gravity and/or fine-tuning in order to have the appropriate behaviour; besides, they lack naturalness and do not exhibit one of the most important properties of theories in modern physics: the existence of internal symmetries. In this paper, we present a vector inflationary model embodied in a $S U(2)$ gauge theory composed of three vector fields over which we make a well supported assumption about their direction, their norm, and their time dependence. Such an assumption allows us to obtain isotropic inflation in a rotationally invariant background if we take into account the homomorphic relation between $S U(2)$ and $O(3)$ groups. In this scenario, we study the dynamics of the vector fields and the conditions to obtain accelerated expansion; we also analyze the evolution of the system in the slow-roll regime and obtain numerical solutions for the equations of motion. Due to the naturalness of the model, the observed numerical results and the generality of the proposed assumption, we conclude that this type of inflation represents well the behaviour of the primordial universe in view of the most recent observations.
\end{abstract}

Key words: Inflationary paradigm, vector fields, non-Abelian gauge field theories, slow-roll inflation.

\section{Introducción}

La base de la cosmología moderna se encuentra en el paradigma inflacionario, ya que éste representa una buena solución para los llamados problemas de la cosmología estándar, a saber: el problema de la planitud, el problema del horizonte y el problema de las reliquias no deseadas (Weinberg, 2008); además, la inflación es crucial para estudiar la generación de estructura a gran escala y la producción de ondas gravitacionales primordiales (BICEP2 Collaboration, Ade, et al., 2014; Lyth \& Liddle, 2009; 
Rubakov, 1982; Starobinsky, 1980; Starobinsky, 1982; Weinberg, 2008). Dicho mecanismo elimina la necesidad de realizar ajustes finos en las condiciones iniciales del universo primitivo, explicando de manera dinámica por qué el universo presenta las características observadas en la actualidad, por ejemplo, el alto grado de isotropía en la radiación cósmica de fondo (Penzias \& Wilson, 1965) y la aparente planitud del universo (Planck Collaboration, Ade, et al., 2013a; WMAP Collaboration, Hinshaw, et al., 2013). En general, el período inflacionario se sitúa antes de la era dominada por la radiación y está caracterizado por presentar expansión acelerada, lo cual implica que también esté dominado por la energía de vacío (Mukhanov, 2005; Weinberg, 2008).

Por estas razones, es indispensable construir modelos del comportamiento primordial del universo que presenten las características fundamentales del mecanismo inflacionario, que tengan fundamentos físicos sólidos y que estén de acuerdo con las observaciones (Planck Collaboration, Ade,et al., 2013a; WMAP Collaboration, Hinshaw, et al., 2013). En general, la dinámica inflacionaria se asocia con la existencia de uno o muchos campos escalares en un espacio con un promedio de partículas de aproximadamente cero y un potencial que controla su comportamiento (Lyth \& Liddle, 2009; Mukhanov, 2005; Weinberg, 2008). Esta primera aproximación produce directamente homogeneidad e isotropía espacial y su tratamiento teórico es bastante sencillo, lo cual permite hacer comparaciones con los datos obtenidos de la observación. Sin embargo, existe un factor que no respalda el modelo inflacionario escalar: recientes mapas de la radiación cósmica de fondo muestran la posible existencia de una dirección privilegiada en el universo (Axelsson, et al., 2013; Cai, Ma, Tang \& Tuo, 2013; Groeneboom, Ackerman, Wehus \& Eriksen, 2010; Groeneboom \& Eriksen, 2009; Kalus, Schwarz, Seikel \& Wiegand, 2013; Kim \& Komatsu, 2013; Paci, et al., 2013; Planck Collaboration, Ade, et al., 2013b; Ramazanov \& Rubtsov, 2014; Singal, 2013; Zhao, Wu \& Zhang, 2013). Este hecho no puede explicarse a partir de campos escalares, lo que implica la necesidad de intentar un tratamiento con cantidades que introduzcan la noción de dirección para poder hacer un estudio que esté de acuerdo con los resultados derivados de la observación. De esta manera, los campos vectoriales adquieren gran importancia en la dinámica inflacionaria, ya que, por su naturaleza, introducen el concepto de dirección espacial.

Mediante los campos vectoriales es posible edificar modelos inflacionarios anisótropos (Maleknejad, Sheikh-Jabbari \& Soda, 2013) siempre y cuando la razón entre el shear $\Sigma$ y

\footnotetext{
* Correspondencia:

Carlos Nieto, carlosfisartnieto@gmail.com

Recibido: 26 de junio de 2013

Aceptado: 16 de abril de 2014
}

el parámetro de expansión global $H$ esté dentro de las cotas observacionales $|\Sigma / H|<0,012$ (Campanelli, Cea, Fogli \& Marrone, 2011; Kalus, Schwarz, Seikel \& Wiegand, 2013); sin embargo, es igualmente factible construir modelos inflacionarios vectoriales isótropos bajo consideraciones muy específicas. La introducción de muchos campos vectoriales con direcciones aleatorias puede en promedio generar inflación isótropa en un fondo rotacionalmente invariante, al igual que la introducción de tres vectores mutuamente ortogonales y de igual norma genera inflación isótropa en el marco de una teoría de gauge U(1) (Armendáriz-Picón, 2007). No obstante, la dinámica inflacionaria en estos modelos cesa muy pronto, llevando a que no se resuelvan los problemas de la cosmología estándar. Una solución para este problema se encuentra a través de modificaciones en la gravedad (Golovnev, Mukhanov \& Vanchurin, 2008). Sin embargo, se encuentra que dichas modificaciones a la gravedad, más el hecho de trabajar con campos vectoriales, puede conllevar a inestabilidades y fantasmas (Golovnev, 2010; Golovnev, 2011; Himmetoglu, Contaldi \& Peloso, 2009a; Himmetoglu, Contaldi \& Peloso, 2009b; Himmetoglu, Contaldi \& Peloso, 2009c). Así, el ajuste especial en la disposición de los campos en ambos modelos, más la necesaria modificación de la gravedad, genera una carencia de naturalidad e implica que estos dos modelos pierdan interés en el contexto científico.

De ahí la necesidad de edificar un modelo que disminuya el número de consideraciones ad hoc y que, por supuesto, involucre campos vectoriales, produzca inflación isótropa (como primera aproximación a un modelo más general), no modifique la gravedad $\mathrm{y}$, como ingrediente adicional, tenga una semejanza con los modelos de la física de partículas (Kane, 1993; Ryder, 1985; Weinberg, 1996). Esto último se fundamenta en el hecho de tener una conexión entre la física durante y después de la inflación. Por ende, se debe tener en cuenta alguna simetría interna del modelo; si bien muchos modelos poseen invariancia ante transformaciones del grupo abeliano $U(1)$, en la naturaleza hay más simetrías del tipo no abeliano, por ejemplo, la simetría ante los grupos $S U(2)$ y SU(3) (Weinberg, 1996).

En el presente artículo se estudia un modelo enmarcado en una teoría invariante de gauge $S U(2)$, ya que así se introducen de forma natural tres campos vectoriales, los cuales generarán la expansión acelerada. Además, a través de una suposición sobre la forma de los campos vectoriales y las propiedades topológicas del grupo $S U(2)$, se logra retener la simetría rotacional del fondo espacial y producir inflación isótropa (Maleknejad \& Sheikh-Jabbari, 2011; Maleknejad \& Sheikh-Jabbari, 2013). Una vez especificada la forma de los campos vectoriales, se construye un lagrangiano que satisfaga la condición necesaria y suficiente para que se produzca una era inflacionaria $(\rho+3 P<0)$; se hace un estudio de la dinámica en el régimen de rodadura lenta, y se encuentra 
una expresión para el monto de expansión en términos de los parámetros de rodadura lenta al inicio de la inflación y las constantes de acoplamiento en la acción del modelo. Por último, se resuelven de manera numérica las ecuaciones de movimiento para encontrar la evolución temporal de las cantidades más importantes del modelo y verificar si el monto de expansión es coherente con lo exigido por las observaciones (Kim \& Murphy, 1985; Lyth \& Liddle, 2009; Weinberg, 2008). De acuerdo con los resultados obtenidos, queda claro que tanto la forma de los campos vectoriales como el lagrangiano del modelo producen de manera exitosa las propiedades buscadas con un número bajo de consideraciones ad hoc, abriendo la posibilidad de hacer estudios sobre recalentamiento, la producción de partículas o la teoría de perturbaciones cosmológicas enmarcados en este modelo inflacionario (Abbott, Farhi \& Wise, 1982; Albrecht, Steinhardt, Turner \& Wilczek, 1982; Allahverdi, Brandenberger, Cyr-Racine \& Mazumdar, 2010; Greene, Kofman, Linde \& Starobinsky, 1997).

\section{Modelo inflacionario: escogencia de los campos vectoriales}

El modelo inflacionario propuesto se construye en el marco de una teoría invariante ante transformaciones del grupo $S U(2)$. Por ende, deben existir tres campos vectoriales $A^{a}{ }_{\mu}$ con los cuales se mantiene el lagrangiano invariante ante dichas transformaciones (Kane, 1993; Ryder, 1985; Weinberg, 1996). Aquí, $a=1,2,3$ corresponden a los índices del álgebra de gauge y $\mu=0,1,2,3$ representan los índices espaciotemporales. En general, las componentes de cada uno de los campos son diferentes los unos de los otros. Sin embargo, debido a la simetría del modelo, es posible fijar un gauge de tal manera que se logren las características deseadas: inflación isótropa en un fondo homogéneo e isótropo descrito por la métrica de Friedmann-Lemaitre-RobertsonWalker (FLRW) (Lyth \& Liddle, 2009; Mukhanov, 2005; Weinberg, 2008) cuya forma es

$$
d s^{2}=-d t^{2}+a^{2}(t) \delta_{i j} d x^{i} d x^{j},
$$

en donde $a(t)$ es el parámetro de expansión global e $i, j$ son los índices a lo largo de una hipersuperficie tridimensional de tipo espacio con tensor métrico descrito por $h_{i j}=a^{2}(t) \delta_{i j}$ en coordenadas cartesianas. El gauge temporal

$$
A_{0}^{a}=0 \text {, }
$$

se manifiesta como una adecuada escogencia debido a que permite hacer una conexión entre los índices del álgebra de gauge y las componentes espaciales de los campos. Al escribir los campos vectoriales en términos de una tétrada ortonormal, se tiene que

$$
A_{\mu}^{a}=\hat{e}_{\mu}^{(\alpha)} A_{(\alpha)}^{a}
$$

en donde $\hat{e}^{(\alpha)}$ son los $(\alpha)$ vectores ortonormales de la tétrada y $A^{a}{ }_{\alpha}$ son las componentes de cada vector $A^{a}{ }_{\alpha}$ a lo largo de los vectores ortonormales. Ahora, usando el gauge de la ecuación (2) y la tétrada

$$
\begin{gathered}
\hat{e}_{\mu}^{(0)}=(1,0,0,0), \hat{e}_{\mu}^{(1)}=(0, a(t), 0,0), \\
\hat{e}_{\mu}^{(2)}=(0,0, a(t), 0), \hat{e}_{\mu}^{(3)}=(0,0,0, a(t)),
\end{gathered}
$$

se tiene que

$$
A_{i}^{a}=\hat{e}^{(j)}{ }_{i} A_{(j)}^{a}
$$

Es aquí donde se introduce la suposición comentada anteriormente, escogiendo los vectores $A^{a}{ }_{(j)}$ de tal forma que estén dirigidos a lo largo de cada vector espacial de la tétrada y que tengan la misma magnitud, es decir,

$$
A_{(j)}^{a}=\psi(t) \delta_{(j)}^{a}
$$

con lo cual se obtiene

$$
A^{a}{ }_{i}=\psi(t) \hat{e}^{a}{ }_{i}=a(t) \psi(t) \delta^{a}{ }_{i} .
$$

Se observa, entonces, que los tres vectores son espaciales, ortogonales y de igual norma; dada la manera en que se definieron los vectores, se pueden identificar los índices espaciales con los índices del álgebra de gauge y, teniendo en cuenta que el grupo $O(3)$ es una imagen homomórfica del grupo $S U(2)$ (Ryder, 1985), se puede ver una rotación de los ejes del espacio interno como una rotación tridimensional. Así, la simetría gauge del modelo es concebida como una simetría de rotación en el espacio y, consecuentemente, el fondo permanece homogéneo e isótropo. Es claro que el ampo $\psi$ transforma como lo hace un campo escalar usual porque se definió en la tétrada ortogonal. Sin embargo, en este caso resulta más conveniente tratar las ecuaciones de evolución con un campo escalar auxiliar $\phi(t)=a(t) \psi(t)$, con lo cual

$$
A^{a}{ }_{i}=\phi(t) \delta^{a}{ }_{i}
$$

Aunque la anterior escogencia luzca como un ajuste fino, se ha demostrado que la expresión $A^{a}{ }_{i}=\phi(t) \delta^{a}{ }_{i}$ es la solución atractor de un modelo inflacionario vectorial anisótropo en un fondo descrito por la métrica de Bianchi tipo I (Maleknejad, Sheikh-Jabbari \& Soda, 2012; Maleknejad, Sheikh-Jabbari \& Soda, 2013). Dicho de otra manera, a pocos e-folds de expansión después del inicio de la inflación, la configuración espacial de los campos evoluciona hasta la forma dada en la ecuación (8). Por ende, la forma propuesta para los campos vectoriales no hace parte de una escogencia fina sino que presenta un soporte físico sólido.

\section{Ecuaciones de campo y de evolución}

Habiendo encontrado la forma de los campos vectoriales necesaria para tener las propiedades deseadas, se requiere ahora definir un lagrangiano que caracterice el modelo inflacionario. Debido a que se trabaja en el marco de una 
teoría invariante de gauge $S U(2)$, el lagrangiano sólo puede tener una dependencia de $A^{a}{ }_{\mu}$ en el tensor de esfuerzos tipo Maxwell $F_{\mu \nu}^{a}$, el cual está definido de la siguiente manera (Kane, 1993; Ryder, 1985; Weinberg, 1996):

$$
F_{\mu \nu}^{a}=\partial_{\mu} A_{\nu}^{a}-\partial_{\nu} A_{\mu}^{a}-g \epsilon_{b c}^{a} A_{\mu}^{b} A_{\nu}^{c},
$$

en donde $g$ es la constante de acoplamiento y $\epsilon_{b c}^{a}$ son las constantes de estructura del grupo $S U(2)$. Entonces, el lagrangiano se escribe en general como $\mathcal{L}=\mathcal{L}\left(F_{\mu \nu}^{a}, g_{\mu \nu}\right)$ y, por ende, las ecuaciones de movimiento y el tensor momentumenergía se escriben de la siguiente manera, respectivamente,

$\mathrm{y}$

$$
D_{\mu} \frac{\partial(\sqrt{-g} \mathcal{L})}{\partial F_{\mu \nu}^{a}}=0
$$

$$
T_{\mu \nu}=-2 \frac{\delta \mathcal{L}}{\delta F_{\sigma}^{a}{ }^{\mu}} F_{\sigma \nu}^{a}+g_{\mu \nu} \mathcal{L},
$$

en donde $g_{\mu \nu}$ es el tensor métrico y $D_{\mu}$ es la derivada covariante en una teoría de gauge $S U(2)$. Para un vector en el espacio interno $B^{a}$, la derivada covariante se define como (Kane, 1993; Ryder, 1985; Weinberg, 1996)

$$
D_{\mu} B^{a}=\partial_{\mu} B^{a}-g \epsilon_{b c}^{a} A_{\mu}^{b} B^{c},
$$

en donde $A^{b}{ }_{\mu}$ es el vector de gauge en el espacio interno. Ahora, si se utiliza la métrica de FLRW y el ansatz definido en la sección anterior, la ecuación (10) se convierte en

$$
\frac{d}{a^{3} d t}\left(a^{3} \frac{\partial \mathcal{L}_{r e d}}{\partial \dot{\phi}}\right)-\frac{\partial \mathcal{L}_{r e d}}{\partial \phi}=0,
$$

en donde $\mathcal{L}_{\text {red }}$ es el lagrangiano reducido obtenido al reemplazar la configuración de los campos vectoriales dada en la ecuación (8) y la métrica de FLRW en $\mathcal{L}=\mathcal{L}\left(F_{\mu \nu}^{a}, g_{\mu \nu}\right)$.

Esta ecuación es idéntica a la ecuación de movimiento de un modelo de inflación isótropa con sólo un campo escalar $\phi$ y un lagrangiano $\mathcal{L}(\phi, \phi, \mathrm{a}(t))$, es decir, el modelo vectorial planteado presenta el tipo de evolución deseado. Por otra parte, se encuentra que las únicas componentes no nulas del tensor momentum-energía del sistema son las correspondientes a la densidad de energía $\rho$ y la presión del fluido $P$

y

$$
\rho=\frac{\partial \mathcal{L}_{r e d}}{\partial \dot{\phi}} \dot{\phi}-\mathcal{L}_{r e d}
$$

$$
P=\frac{\partial\left(a^{3} \mathcal{L}_{\text {red }}\right)}{\partial a^{3}}
$$

confirmándose de esta manera que el universo compuesto por los campos definidos en la ecuación (8) puede modelarse como un fluido perfecto. Es importante anotar que los resultados mostrados son válidos para cualquier lagrangiano $\mathcal{L}=\mathcal{L}\left(F_{\mu \nu}^{a}, g_{\mu \nu}\right)$ siempre y cuando se haga uso de la suposición dada en la ecuación (8) y la métrica de FLRW. Por lo tanto, el gauge escogido y la forma utilizada para las componentes espaciales de los campos resultan satisfactorias, ya que reproducen las propiedades geométricas requeridas independientemente del lagrangiano escogido para el modelo. Sin embargo, si se quiere producir una era caracterizada por expansión exponencial se debe dar forma a $\mathcal{L}=\mathcal{L}\left(F_{\mu \nu}^{a}, g_{\mu \nu}\right)$.

\section{El lagrangiano del modelo}

Como se afirmó en la sección anterior, el lagrangiano debe incluir términos que incluyan a $F_{\mu \nu}^{a}$ para que la teoría sea invariante de gauge. El término más sencillo, que es a la vez invariante de gauge y de Lorentz, es $-\frac{1}{4} F_{\mu \nu}^{a} F_{a}^{\mu \nu}$ (término de Yang-Mills). Este primer término tiene una forma análoga al término cinético del lagrangiano electromagnético y produce una ecuación de estado $P=\rho / 3$ que caracteriza un fluido compuesto por radiación, lo cual es bueno, puesto que se espera que después de la inflación se tenga una era dominada por la radiación. No obstante, es indispensable que a través del lagrangiano escogido se satisfaga la condición necesaria y suficiente para tener expansión acelerada (Lyth \& Liddle, 2009; Mukhanov, 2005; Weinberg, 2008)

$$
\rho+3 P<0
$$

Se debe tener, entonces, un término adicional que lleve a dicha relación, al menos durante los tiempos tempranos. Una opción es aumentar el grado de $F_{\mu \nu}^{a}$ dentro del lagrangiano, pero esto llevaría a una expresión algebraicamente complicada para la condición en la ecuación (16). Sin embargo, si se multiplica el producto $F_{\mu \nu}^{a} F_{a}^{\mu \nu}$ por el tensor $\epsilon^{\mu \nu \lambda \sigma}$ asociado al símbolo de Levi-Civita, se obtienen resultados atractivos y se encuentra una dinámica interesante entre este nuevo término y el término de Yang-Mills. Entonces, se propone el nuevo término para el lagrangiano

$$
\left(\epsilon^{\mu \nu \lambda \sigma} F_{\mu \nu}^{a} F_{\lambda \sigma}^{a}\right)^{2}
$$

en donde el tensor contravariante $\epsilon^{\mu \nu \lambda \sigma}$ se define a través de la densidad tensorial contravariante $\varepsilon^{\mu \nu \lambda \sigma}$ (símbolo de LeviCivita) y el determinante de la métrica, así:

$$
\epsilon^{\mu \nu \lambda \sigma}=\frac{\varepsilon^{\mu \nu \lambda \sigma}}{\sqrt{-g}} .
$$

En síntesis, el lagrangiano total sería

$$
\mathcal{L}=-\frac{1}{4} F_{\mu \nu}^{a} F_{a}^{\mu \nu}+\frac{\kappa}{384}\left(\epsilon^{\mu \nu \lambda \sigma} F_{\mu \nu}^{a} F_{\lambda \sigma}^{a}\right)^{2},
$$

en donde $\kappa$ es un parámetro libre y 384 es un número entero necesario para facilitar el tratamiento de las ecuaciones dinámicas. La idea de postular el lagrangiano en la ecuación (19) es tener un balance dinámico entre los dos términos, de tal manera que uno de ellos sea el que genere y mantenga la era inflacionaria y el otro el que le dé fin en el momento justo. 
Una vez definido el lagrangiano, se procede a encontrar la ecuación de evolución de los campos y las componentes no nulas del tensor momentum-energía. Para esto, se hace indispensable encontrar $\mathcal{L}_{\text {red }}$ y usar las ecuaciones (13), (14) y (15). Tomando la configuración de $A_{i}^{a}$ presentada en la ecuación (8) y la métrica de FLRW, se obtienen las componentes de $F_{\mu \nu}^{a}$

$$
F_{00}^{a}=0, \quad F_{0 i}^{a}=\dot{\phi} \delta_{i}^{a}, \quad F_{i j}^{a}=-g \phi^{2} \epsilon_{i j}^{a},
$$

y el producto

$$
\epsilon^{\mu \nu \lambda \sigma} F_{\mu \nu}^{a} F_{\lambda \sigma}^{a}=4 \epsilon^{i j k 0} g \dot{\phi} \phi^{2} \varepsilon^{k}{ }_{i j} .
$$

De esta manera, el lagrangiano en la ecuación (19) se convierte en

$$
\begin{aligned}
\mathcal{L}= & \frac{1}{2} \frac{\dot{\phi}^{2}}{a^{2}} \delta^{a}{ }_{i} \delta_{a i}-\frac{1}{4} \frac{g^{2} \phi^{4}}{a^{4}} \varepsilon_{a k j} \varepsilon_{a k j} \\
& +\frac{\kappa}{24} \epsilon^{i j k 0} \epsilon^{m n p 0} g^{2} \dot{\phi}^{2} \phi^{4} \varepsilon_{i j k} \varepsilon_{m n p}
\end{aligned}
$$

en donde se utiliza la definición de $\epsilon^{\mu \nu \lambda \sigma}=\varepsilon^{\mu \nu \lambda \sigma} / \sqrt{-g} \mathrm{y}$ se hacen las debidas sumatorias para encontrar, finalmente,

$$
\mathcal{L}_{\text {red }}=\frac{3}{2}\left[\frac{\dot{\phi}^{2}}{a^{2}}-\frac{g^{2} \phi^{4}}{a^{4}}+\kappa \frac{g^{2} \dot{\phi}^{2} \phi^{4}}{a^{6}}\right] .
$$

Entonces, la densidad de energía y la presión del fluido son

$$
\begin{gathered}
\rho=\frac{3}{2}\left(\frac{\dot{\phi}^{2}}{a^{2}}+\frac{g^{2} \phi^{4}}{a^{4}}+\kappa \frac{g^{2} \dot{\phi}^{2} \phi^{4}}{a^{6}}\right), \\
P=\frac{1}{2}\left(\frac{\dot{\phi}^{2}}{a^{2}}+\frac{g^{2} \phi^{4}}{a^{4}}\right)-\frac{3}{2} \kappa \frac{g^{2} \dot{\phi}^{2} \phi^{4}}{a^{6}},
\end{gathered}
$$

de donde se puede hallar la ecuación de Friedmann $H^{2}=\rho / 3$ para este modelo

$$
H^{2}=\frac{1}{2}\left(\frac{\dot{\phi}^{2}}{a^{2}}+\frac{g^{2} \phi^{4}}{a^{4}}+\kappa \frac{g^{2} \dot{\phi}^{2} \phi^{4}}{a^{6}}\right),
$$

al igual que su derivada temporal

$$
\dot{H}=-\left(\frac{\dot{\phi}^{2}}{a^{2}}+\frac{g^{2} \phi^{4}}{a^{4}}\right) .
$$

Por otra parte, la ecuación de evolución de los campos adquiere la forma

$$
\begin{aligned}
& \left(1+\kappa \frac{g^{4} \phi^{4}}{a^{4}}\right) \ddot{\phi}+\left(1+\kappa \frac{\dot{\phi}^{2}}{a^{2}}\right) \frac{2 g^{2} \phi^{3}}{a^{2}} \\
& +\left(1-3 \kappa \frac{g^{2} \phi^{4}}{a^{4}}\right) \dot{\phi} H=0 .
\end{aligned}
$$

Como se precisó al principio, se debe estudiar la condición necesaria y suficiente para que se dé la inflación. Para esto, se definen dos cantidades asociadas a la densidad de energía

$$
\begin{gathered}
\rho_{Y M}=\frac{3}{2}\left(\frac{\dot{\phi}^{2}}{a^{2}}+\frac{g^{2} \phi^{4}}{a^{4}}\right), \\
\rho_{\kappa}=\frac{3}{2} \kappa \frac{g^{2} \phi^{4} \dot{\phi}^{2}}{a^{6}},
\end{gathered}
$$

con las cuales la ecuación de Friedmann (26) y su derivada temporal (27) se reducen a

$$
\begin{gathered}
H^{2}=\frac{1}{3}\left(\rho_{Y M}+\rho_{\kappa}\right), \\
\dot{H}=-\frac{2}{3} \rho_{Y M} .
\end{gathered}
$$

Se dice que $\rho_{Y M}$ es la densidad de energía asociada al término de Yang-Mills y $\rho_{\kappa}$ es la densidad de energía asociada al término $\kappa$. Por ende, la densidad total de energía y la presión se escriben como

$$
\begin{gathered}
\rho=\rho_{Y M}+\rho_{\kappa^{\prime}} \\
P=\frac{1}{3} \rho_{Y M}-\rho_{\kappa} .
\end{gathered}
$$

Así, la suma $\rho+3 P$ es

$$
\rho+3 P=2\left(\rho_{Y M}-\rho_{\mathrm{K}}\right),
$$

de donde se concluye que la condición para tener expansión acelerada es

$$
\rho_{\mathrm{K}}>\rho_{Y M}
$$

Es decir, se espera que al inicio de la evolución del universo la densidad de energía asociada al término $\kappa$ domine sobre la densidad de energía asociada al término de Yang-Mills. Después de cierto tiempo, $\rho_{\kappa}$ debe decrecer con respecto a $\rho_{Y M}$ de tal manera que la inflación termine y empiece la era dominada por la radiación. Con la solución numérica de las ecuaciones, se debe analizar la condición en la ecuación (36) $\mathrm{y}$, de esta manera, garantizar parte del éxito del modelo.

\section{Inflación del tipo rodadura lenta}

Una vez obtenidas las ecuaciones de movimiento y las ecuaciones de Einstein, se hace un estudio dinámico en el régimen de rodadura lenta. Este tipo de inflación es importante por dos razones: primero, permite obtener un tiempo apreciable de expansión acelerada, de tal manera que se puedan resolver los problemas de la cosmología estándar (Lyth \& Liddle, 2009; Mukhanov, 2005; Weinberg, 2008) y, segundo, estaría de acuerdo con las observaciones sobre la invariancia de escala en la perturbación primordial en la curvatura (Planck Collaboration, Ade, et al., 2013a; WMAP Collaboration, Hinshaw, et al., 2013). Para empezar, se introducen los parámetros que caracterizan la inflación de tipo rodadura lenta para un modelo inflacionario escalar, es decir, $\varepsilon$ y $\eta$ así:

$$
\varepsilon=-\frac{\dot{H}}{H^{2}}, \quad|\eta-\varepsilon|=\left|-\frac{\ddot{\phi}}{H \dot{\phi}}\right|,
$$


en donde $H=\dot{a} / a$ es el parámetro de Hubble y $\phi$ es el campo escalar del modelo (Lyth \& Liddle, 2009). Sin embargo, para hacer el estudio más general, estos parámetros se escribirán sólo en términos de $H$ y sus derivadas como sigue ${ }^{1}$

$$
\varepsilon \equiv-\frac{\dot{H}}{H^{2}}, \quad \eta \equiv \frac{\ddot{H}}{2 \dot{H} H},
$$

de donde se obtiene la siguiente relación entre ellos:

$$
\eta=\varepsilon-\frac{\dot{\varepsilon}}{2 \varepsilon H}
$$

Ahora, debido a que los parámetros estándar $\varepsilon$ y $\eta$ fueron escritos en términos de $H$, se requiere un nuevo parámetro que muestre de manera explícita el comportamiento del campo escalar $\psi$ del modelo. Por esto, además de asegurarse de tener una variación pequeña de $H$, se definirá un parámetro que caracterice la evolución del campo $\psi$ durante la inflación:

$$
\delta \equiv-\frac{\dot{\psi}}{\psi H}
$$

Así, con el nuevo parámetro se completa el conjunto de parámetros de rodadura lenta del modelo inflacionario vectorial de gauge no abeliano:

$$
\varepsilon \equiv-\frac{\dot{H}}{H^{2}}, \quad \eta \equiv-\frac{\ddot{H}}{2 \dot{H} H}, \quad \delta \equiv-\frac{\dot{\psi}}{\psi H} .
$$

Antes de encontrar las expresiones para $\varepsilon, \eta$ y $\delta$ concernientes al modelo propuesto, se examinarán las condiciones que deben existir sobre estos parámetros y las implicaciones sobre las cantidades dinámicas como la densidad de energía. Se sabe que $\varepsilon,|\eta| \ll 1$ para tener una dinámica de rodadura lenta; así, usando la expresión para $\varepsilon$ se encuentra que

$$
\varepsilon=\frac{2 \rho_{Y M}}{\rho_{Y M}+\rho_{\kappa}} \ll 1,
$$

de donde se deduce que $\rho_{\kappa}$ debe dominar sobre $\rho_{Y M}$ para tener el comportamiento deseado, es decir,

$$
\rho_{\kappa} \gg \rho_{Y M}
$$

Por lo tanto, se espera que la densidad de energía asociada al término $\kappa$ sea mucho mayor que la densidad de energía asociada al término de Yang-Mills en los tiempos tempranos. Una desigualdad similar se encontró cuando se analizaba la condición para tener una era de expansión acelerada; en ese caso, se había observado que $\rho+3 P<0$ implicaba $\rho_{\kappa}>$ $\rho_{Y M^{M}}$. Sin embargo, aquí se exige que $\rho_{\mathrm{K}}$ sea mucho mayor que $\rho_{Y M}$, por lo que se concluye que $\rho_{\kappa} \gg \rho_{Y M}$ es una condición suficiente mas no necesaria para que se produzca

\footnotetext{
1 Se puede mostrar que las expresiones en la ecuación (38) aplicadas a un modelo inflacionario compuesto por un campo escalar se reducen a las expresiones en la ecuación (37).
}

una era de expansión acelerada. En resumen, se tiene que, al inicio de la inflación, la evolución de los campos debe ser tal que $\rho_{\kappa} \gg \rho_{Y M}$ para tener dinámica de rodadura lenta; luego, después de un tiempo de evolución, $\rho_{Y M}$ aumentará más rápidamente que $\rho_{\mathrm{k}}$, de tal manera que cuando $\rho_{Y M} \sim \rho_{\mathrm{k}}$, la inflación del tipo rodadura lenta finalice, y cuando $\rho_{Y M}$ $>\rho_{\mathrm{k}}$, la era de expansión acelerada llegue a su fin habiendo resuelto los problemas de la cosmología estándar.

A continuación se procede a encontrar las expresiones para los parámetros de rodadura lenta y el monto de expansión $N_{e}$ (Lyth \& Liddle, 2009; Mukhanov, 2005; Weinberg, 2008). Con la expresión para $\varepsilon$ en la ecuación (38) y las expresiones para $\rho_{Y M}$ y $\rho_{\kappa}$ de las ecuaciones (29) y (30), se obtiene la igualdad

$$
\varepsilon=2-2 \frac{\rho_{\kappa}}{\rho_{Y M}+\rho_{\kappa}}=2-\frac{\kappa g^{2} \phi^{4} \dot{\phi}^{2}}{a^{6} H^{2}},
$$

la cual puede ser convertida en

$$
\varepsilon=2-\kappa^{2} \psi^{6}(1-\delta)^{2},
$$

teniendo en cuenta que $\psi=\phi / \mathrm{a}$ y $\delta=1-\dot{\phi} / \mathrm{H} \phi$. A partir de la ecuación (39) y del resultado anterior, se encuentra una expresión para $\eta$ en términos de los parámetros $\varepsilon$ y $\delta$

$$
\eta=\varepsilon-(2-\varepsilon)\left[\frac{\dot{\delta}}{H(1-\delta) \varepsilon}+\frac{3 \delta}{\varepsilon}\right] .
$$

Ahora, imponiendo las condiciones usuales sobre $\varepsilon$ y $\eta$ (i.e. $\varepsilon,|\eta| \ll 1$ ) (Lyth \& Liddle, 2009; Mukhanov, 2005; Weinberg, 2008), se encuentra el orden de magnitud de $\delta$, es decir, la condición de variación lenta del campo físico $\psi$ durante la inflación. A través de la ecuación (39) y las condiciones señaladas anteriormente, se encuentra que se debe cumplir

$$
\dot{\varepsilon} \sim H \varepsilon^{2}, \eta \sim \varepsilon,
$$

para evitar ajustes finos en las cantidades $\varepsilon$ y $\eta$. Con esto se deduce, a través de la ecuación (46), que el orden de magnitud del parámetro $\delta$ es

$$
\delta \sim \varepsilon^{2},
$$

lo que evidencia que $\psi$ varía lentamente durante la inflación.

Para encontrar la forma funcional de los parámetros $\varepsilon, \eta$ y $\delta$ en el régimen de rodadura lenta se usa la condición (43), por lo cual la ecuación de Friedmann (26) se convierte en

$$
H^{2} \simeq \frac{1}{3} \rho_{\kappa}
$$

y el parámetro $\varepsilon$ toma la forma

$$
\varepsilon \simeq \frac{\dot{\phi}^{2} / a^{2}}{H^{2}}+\frac{g^{2} \phi^{4} / a^{4}}{H^{2}}
$$


o, en términos de $\psi$,

$$
\varepsilon \simeq \frac{2}{\kappa g^{2} \psi^{4}}+\frac{g^{2} \psi^{4}}{H^{2}}
$$

Por otra parte, de la ecuación (44) se tiene que durante el período de rodadura lenta

$$
\kappa=\frac{2}{g^{2} \psi^{6}},
$$

$\mathrm{y}$, por ende, el parámetro $\varepsilon$ se convierte en

$$
\begin{aligned}
& \varepsilon \simeq \psi^{2}+\frac{g^{2} \psi^{4}}{H^{2}}, \\
& \varepsilon \simeq \psi^{2}(\gamma+1),
\end{aligned}
$$

definiendo la cantidad $\gamma=\psi^{2} g^{2} / H^{2}$. Siguiendo la misma línea de análisis, se puede encontrar una expresión para el parámetro $\eta$ en el régimen de rodadura lenta; por medio de la ecuación (39) y la forma de $\varepsilon$ mostrada en (54) se encuentra

$$
\eta \simeq \psi^{2}
$$

Por último, para hallar la ecuación que relaciona $\delta$ con el campo $\psi$, se usan las ecuaciones (46), (54) y (55), y se obtiene

$$
\left[\frac{\dot{\delta}}{H \delta}+3\right] \simeq \frac{\gamma}{2(\gamma+1)} \varepsilon^{2},
$$

de donde se observa que si $\dot{\psi} / H \psi \ll 1$ entonces $\delta \simeq$ $\frac{\gamma}{6(\gamma+1)} \varepsilon^{2} \simeq \frac{\gamma(\gamma+1) \psi^{4}}{6}$, lo cual es consecuente con el análisis hecho acerca de los órdenes de magnitud de cada parámetro (véase la ecuación (48).

Una de las motivaciones para el uso de las condiciones de rodadura lenta es el monto de inflación $N_{e}$ que indica cuánto se expandió el universo en los tiempos tempranos (Lyth \& Liddle, 2009; Mukhanov, 2005; Weinberg, 2008). Dicho valor es importante porque determina si los problemas clásicos de la cosmología estándar se resuelven satisfactoriamente (Kim \& Murphy, 1985; Lyth \& Liddle, 2009; Weinberg, 2008). Por ende, es interesante encontrar una expresión para el monto de expansión en términos de los parámetros de rodadura lenta del modelo propuesto. Para esto, se tiene én cuenta que $\delta \equiv-\psi / \mathrm{H} \psi$ y $\delta \sim \varepsilon^{2}$,ya que esto permite establecer que $\gamma \mathrm{H}^{2}$ permanece constante durante el régimen de rodadura lenta y, por lo tanto, se establecen las relaciones

$$
\frac{\varepsilon}{\varepsilon_{i}} \simeq \frac{\gamma+1}{\gamma_{i}+1}, \quad \frac{\gamma}{\gamma_{i}} \simeq \frac{H^{2}}{H_{i}^{2}},
$$

en donde el subíndice $i$ indica el inicio de la inflación. Ahora, considerando que al final de la inflación del tipo rodadura lenta $\varepsilon=1$, se llega a

$$
\gamma \simeq \frac{\gamma_{i}+1}{\varepsilon_{i}}, \quad \frac{H_{i}^{2}}{H^{2}} \simeq \frac{\gamma_{i}}{\gamma_{i}+1} \varepsilon_{i},
$$

de donde es posible encontrar el monto de expansión acelerada. De manera general, el monto de expansión está definido de la siguiente manera:

$$
N_{e} \equiv \int_{t_{i}}^{t_{f}} H d t
$$

en donde $t_{i}$ y $t_{f}$ son los tiempos inicial y final de la inflación. Sin embargo, debido a que no se conoce $H$ como función del tiempo, se hace necesario hacer el cambio de variable $d t=$ $d H / \dot{H}$, puesto que de esta manera se tiene

$$
N_{e}=-\int_{H_{i}}^{H_{f}} \frac{d H}{H \varepsilon} .
$$

En la anterior expresión se hace otro cambio de variable $d H=-\frac{H^{3}}{2 q^{2} \psi^{2}} d \gamma$ para encontrar una expresión integrable

$$
N_{e} \simeq-\frac{1}{2 \psi^{2}} \int_{\gamma_{i}}^{\gamma_{f}} \frac{-1 / \gamma^{2}}{(\gamma+1) / \gamma} d \gamma
$$

a partir de la cual se encuentra el monto de expansión para el modelo de estudio

$$
N_{e} \simeq \frac{\gamma_{i}+1}{2 \varepsilon_{i}} \ln \left[\frac{\gamma_{i}+1}{\gamma_{i}}\right]
$$

Se aprecia que $N_{e}$ depende de los valores iniciales de $\gamma$ y $\varepsilon$, y no de su evolución temporal a lo largo de la inflación. Debido a que $\gamma$ y $\varepsilon$ dependen de $\psi$ y $H$, es claro que para resolver los problemas de la cosmología estándar se deben fijar los valores iniciales de $\psi$ y $H$, así como el valor de las constantes $g$ y $\kappa$; el ajuste de estas cantidades se hace a partir de las cotas observacionales sobre la escala de energía durante la inflación. Una vez encontrado el valor de las condiciones iniciales de $\gamma$ y $\varepsilon$, se reemplazan en la ecuación (62) para comprobar que el valor de $N_{e}$ satisface la cota inferior $(N \gtrsim 60)$ (Kim \& Murphy, 1985; Lyth \& Liddle, 2009; Weinberg, 2008).

\section{Solución numérica de las ecuaciones de evolución}

En esta sección se muestra la solución numérica del sistema de ecuaciones dinámicas (26), (27) y (28). Se espera que las variables físicas presenten las características apropiadas durante la inflación: pequeña variación del campo escalar $\psi$ y subsecuente oscilación, dominio de $\rho_{\kappa}$ sobre $\rho_{Y M}$ al principio de la evolución temporal, un valor de $\varepsilon \ll 1$ y una pequeña variación durante la inflación del tipo rodadura lenta, y el monto de expansión $N_{e}$ superior a 60 al final de la inflación. Debido a que las ecuaciones (26), (27) y (28) están escritas en términos del factor de expansión, debe hacerse una modificación para que éstas queden escritas en términos de $H$ (cantidad física medible). Además, como se busca la evolución temporal de $\psi$, es mejor escribir todas las ecuaciones en términos de $\psi$ en lugar de $\phi$.

Así, el nuevo conjunto de ecuaciones es

$$
\begin{gathered}
2 H^{2}=(\dot{\psi}+\psi \mathrm{H})^{2}\left(1+\kappa g^{2} \psi^{4}\right)+g^{2} \psi^{4}, \\
\dot{H}=-(\dot{\psi}+\psi \mathrm{H})^{2}-g^{2} \psi^{4}, \\
\left(1+\kappa g^{2} \psi^{4}\right)\left(\ddot{\psi}+2 \dot{\psi} \mathrm{H}+\psi \dot{H}+\psi \mathrm{H}^{2}\right) \\
+\left(1+\kappa(\dot{\psi}+\psi \mathrm{H})^{2}\right) 2 g^{2} \psi^{3} \\
+\left(1-3 \kappa g^{2} \psi^{4}\right)(\dot{\psi}+\psi \mathrm{H}) H=0 .
\end{gathered}
$$


En cuanto a las condiciones iniciales y los parámetros libres del modelo, estos se escogen de acuerdo a las cotas sobre la escala de energía durante la inflación fijadas por los resultados de WMAP y Planck (Planck Collaboration, Ade, et al., 2013a; WMAP Collaboration, Hinshaw, et al., 2013). Entonces, para encontrar la solución del sistema de ecuaciones se toman las condiciones $\psi_{i}=0,035 M_{p l} \mathrm{y}_{\psi_{i}}=-1 \times 10^{-10} M_{p l}, H_{i}=$ $3,4 \times 10^{-5} M_{p l}, g=2,5 \times 10^{-3}$ y $\kappa=1,733 \times 10^{14} M_{p l}^{-4}$, en donde $M_{p l}$ es la masa reducida de Planck, la cual se define como $M_{p l} \equiv\left(8 \pi \mathrm{G}_{N}\right)^{-1 / 2}=2,43 \times 10^{18} \mathrm{GeV}$. Dado que los resultados son numéricos, resulta interesante observar los gráficos de evolución temporal de las cantidades físicas más importantes: $\psi$ (campo escalar), $\varepsilon$ (parámetro de rodadura lenta) y $N_{e}$ (monto de expansión). Además, se grafica la relación $\rho_{\kappa} / \rho_{Y M}$ para observar cómo evoluciona $\rho_{\kappa}$ con respecto a $\rho_{Y M}$.

En la figura (1) se aprecia claramente que $\psi$ varía muy lentamente en los tiempos tempranos, conduciendo a una era de inflación del tipo rodadura lenta en consecuencia con la condición encontrada líneas atrás, es decir, $\delta \ll 1$; luego de cierto tiempo de evolución, el campo empieza a variar hacia un período de oscilación en el cual el campo $\psi$ decaerá en otras partículas, dando inicio al proceso de recalentamiento (Greene, Kofman, Linde \& Starobinsky, 1997; Lyth \& Liddle, 2009). En la figura (2) se observa que $\varepsilon$ empieza su evolución con un valor muy pequeño $(\varepsilon \ll 1)$ y varía lentamente durante cierto tiempo; luego, en el mismo instante en que $\psi$ empieza a variar, se observa un aumento en el valor de $\varepsilon$ hasta que llega a ser 1 debido a que $\rho_{\kappa} \sim \rho_{Y}$ ${ }_{M} \mathrm{y}$ la inflación del tipo rodadura lenta llega a su fin (en este momento la ecuación de estado es $\rho+3 P=0$ ); finalmente, $\varepsilon$ llega a su valor máximo $(\varepsilon=2) \mathrm{y}$, en este momento, $\rho_{Y M} \sim$ $\rho_{\kappa^{\prime}}$, por lo cual finaliza la era inflacionaria y empieza la época dominada por la radiación.

Estas últimas conclusiones están respaldadas por el comportamiento de $\rho_{\kappa} / \rho_{Y M}$ en función del tiempo, como se muestra en la figura (3); allí se evidencia que en los tiempos tempranos, $\rho_{\kappa}$ es mayor que $\rho_{Y M}$, al menos en dos órdenes de magnitud (lo que hace posible la inflación del tipo rodadura lenta); sin embargo, $\rho_{\kappa}$ decae rápidamente, de tal manera que al final de la inflación $\rho_{Y M}$ domina notablemente; así, es claro que las condiciones para la inflación $\left(\rho_{\kappa}>\rho_{Y M}\right)$ y para la inflación del tipo rodadura lenta $\left(\rho_{\kappa} \gg \rho_{Y M}\right)$ se cumple satisfactoriamente. Por último, en la figura (4) se muestra el comportamiento del monto de expansión en función del tiempo; allí se observa que durante la inflación, $N_{e}$ aumenta rápidamente hasta conseguir el valor requerido para poder resolver los problemas de la cosmología estándar (Kim \& Murphy, 1985; Lyth \& Liddle, 2009; Weinberg, 2008); luego, su aumento es lento debido a que la expansión del universo deja de ser exponencial.

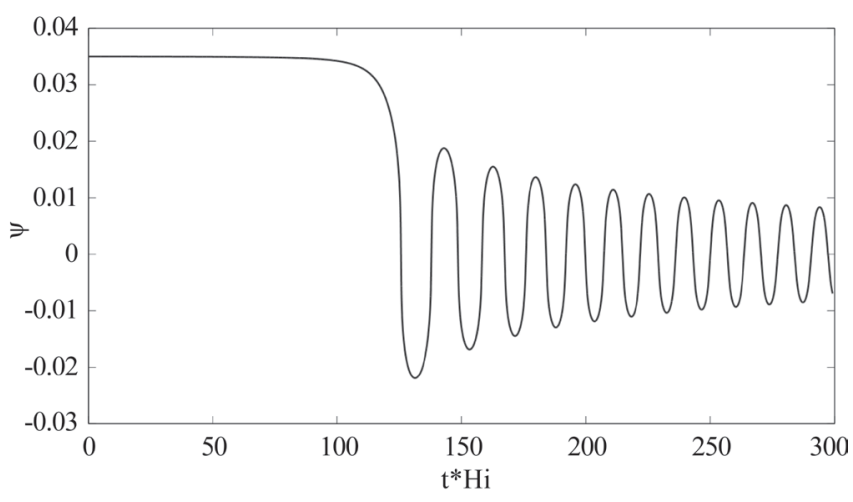

Figura 1. Evolución obtenida para $\psi$ en función de $t H_{i}$

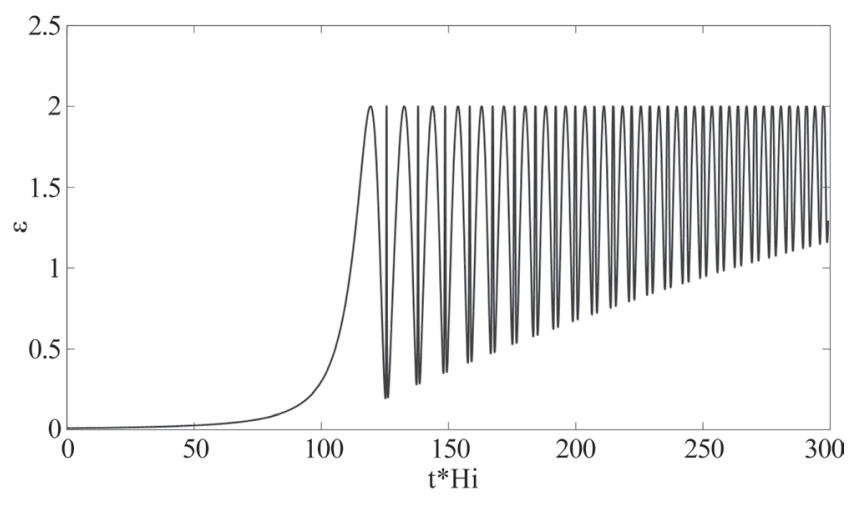

Figura 2. Evolución encontrada para $\varepsilon$ en función de $t H_{i}$

Con estos resultados gráficos se muestra que hay coherencia entre el estudio analítico del modelo durante la inflación del tipo rodadura lenta y el comportamiento de las variables físicas encontrado a partir de la solución numérica de las ecuaciones de movimiento. De manera natural, el modelo satisface la condición necesaria y suficiente para obtener un comportamiento de expansión acelerada y, además, presenta las propiedades representativas de la inflación del tipo rodadura lenta. Por lo tanto, se evidencia la relevancia de asumir las condiciones de rodadura lenta, puesto que ellas permiten tener una idea acerca del comportamiento de las variables físicas en este momento, así como conocer el monto de expansión sin necesidad de conocer el comportamiento de $N_{e}$ a lo largo del tiempo: basta simplemente con usar la expresión (62) y las condiciones iniciales de $\varepsilon$ y $\gamma$ para conocer su valor. En síntesis, se hace claro que el aspecto clave del modelo recae en la dinámica combinada del término de Yang-Mills y el término $\kappa$, ya que de esta manera se generan las características deseadas (por ejemplo, $\rho+3 P$ $<0)$ y su acción conjunta hace que la expansión se prolongue durante el tiempo suficiente para que $N_{e}$ alcance el valor mínimo requerido. 


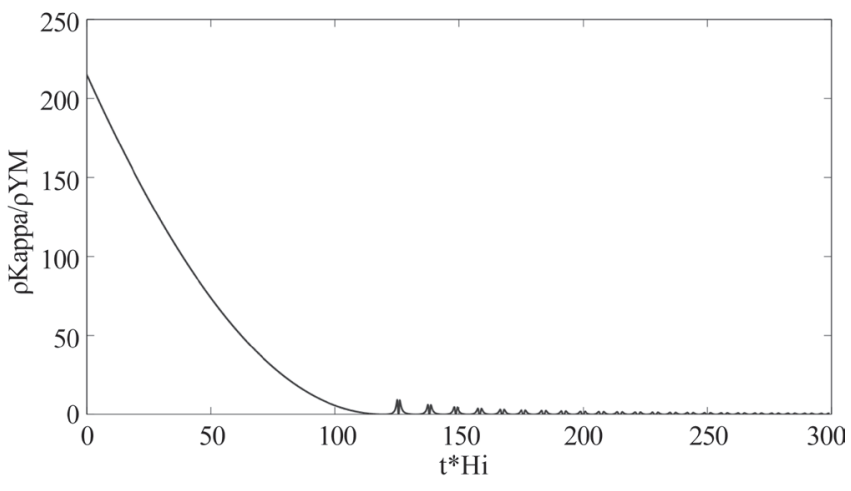

Figura 3. Evolución obtenida para $\rho_{\kappa} / \rho_{Y M}$ en función de $t H_{i}$

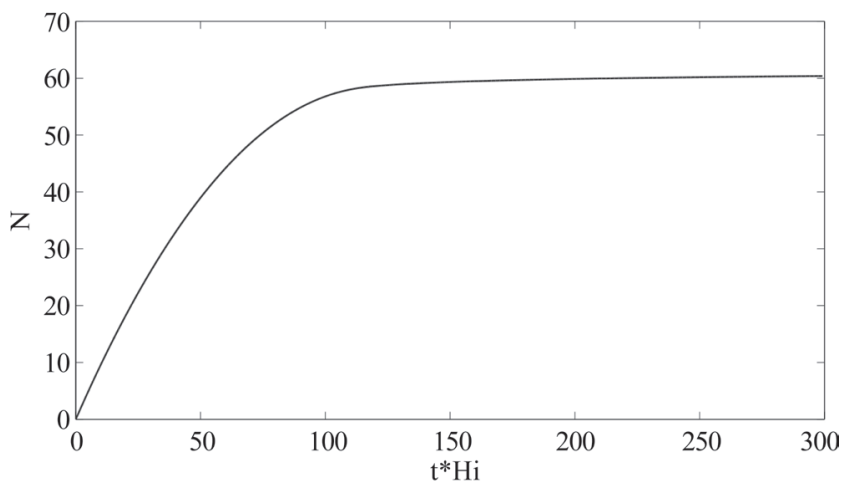

Figura 4. Evolución obtenida para el monto de expansión $N$ en función de $t H_{i}$. Aquí se puede ver que $N$ alcanza el valor cercano a la cota inferior conocida $\left(N_{e} \gtrsim 60\right)$, resolviendo así los problemas clásicos de la cosmología estándar.

\section{Conclusiones}

En este artículo se presentó la construcción de un modelo inflacionario vectorial en un marco de teorías de gauge no abelianas (Maleknejad \& Sheikh-Jabbari, 2011; Maleknejad \& Sheikh-Jabbari, 2013) con el objetivo de acercar la física durante la inflación con la física conocida en el modelo estándar de partículas elementales, en la que la invariancia ante el grupo $S U(2)$ caracteriza las interacciones débiles.

En dicha presentación se aprecia que la forma escogida para los campos vectoriales es crucial debido a que produce expansión isótropa en un fondo rotacionalmente invariante (gracias, igualmente, al hecho de trabajar en el marco de una teoría invariante de gauge $S U(2)$ ). Por otra parte, a pesar de que el término cinético canónico del lagrangiano usual para una teoría de gauge no abeliana (lagrangiano de Yang-Mills) no sirve para generar una época inflacionaria, se encuentra una adecuada modificación, introduciendo un término cuártico en $F_{\mu \nu}{ }^{a}$ multiplicado por un tensor de Levi-Civita, que permite obtener de manera satisfactoria las propiedades deseadas ( $\rho$
$+3 P<0$ ). Como se observa en la solución numérica de las ecuaciones, el término $\kappa$, además de contribuir a la ecuación de estado con un signo menos, presenta un mayor efecto sobre la dinámica inflacionaria al inicio de la evolución temporal, haciendo que la variación del parámetro $\varepsilon$ sea lenta al inicio de la inflación. La dinámica conjunta de $\rho_{\kappa}$ y $\rho_{Y M}$ es esencial para dar inicio y final a la inflación, ya que se espera que la expansión acelerada no sea eterna sino que tenga un final y dé paso a las demás eras existentes en la historia del universo. El estudio de la inflación del tipo rodadura lenta permitió tener una idea acerca del comportamiento de las variables físicas relevantes y, a través de él, se obtuvo una expresión para el monto de inflación expresado en la ecuación (62), a partir de la cual se encuentra que $N_{e} \sim 60$ usando los valores iniciales tomados en la solución numérica. Es importante anotar que la solución numérica de las ecuaciones no se obtuvo teniendo en cuenta aproximaciones de rodadura lenta ni con suposiciones adicionales; los resultados observados surgieron del conjunto completo de ecuaciones y, como se dijo anteriormente, de la dinámica de los dos términos del lagrangiano propuesto. Otro aspecto importante es la escala de energía encontrada en el modelo, la cual es del orden de la escala de energía de gran unificación $\left(\sim 10^{16} \mathrm{GeV}\right)$ (BICEP2 Collaboration, Ade, et al., 2014; Dine, 2007), lo que permite tener una idea de la física en esta época del universo y su relación con la física de partículas conocida, planteando así la posibilidad de construir modelos de recalentamiento en el marco de la inflación vectorial basados en teorías de gauge no abelianas. Se concluye, entonces, que el modelo presenta de manera natural todas las características propias de un modelo inflacionario con expansión isótropa sin modificación de la gravedad ni ajustes finos ad hoc, haciendo de éste un buen descriptor del comportamiento del universo primordial.

\section{Agradecimientos}

Y.R. cuenta con el apoyo financiero de la Fundación para la Promoción de la Investigación y la Tecnología del Banco de la República mediante proyecto de investigación número 3025 CT-2012-02, de la DIEF de Ciencias (UIS) mediante proyecto de investigación número 5709, de la VCTI (UAN) mediante proyecto de investigación número 20131041, y de Colciencias mediante proyecto de investigación número RC 0384-2013. Y.R. y C.M.N. cuentan con el apoyo financiero de Colciencias - ECOS-NORD mediante proyecto de investigación número RC 0899-2012. C.M.N. cuenta con el apoyo financiero de la UIS a través de su programa de becas de posgrado.

\section{Conflicto de interés}

Los autores declaran que no tienen conflicto de interés.

\section{Bibliografía}

Abbott L., Farhi E., Wise M. B., 1982. Particle production in the new inflationary cosmology. Phys. Lett. 117B: 29. 
Albrecht A., Steinhardt P. J., Turner M. S., Wilczek F., 1982. Reheating an inflationary universe. Phys. Rev. Lett. 48: 1437.

Allahverdi R., Brandenberger R., Cyr-Racine F.-Y., Mazumdar A., 2010. Reheating in inflationary cosmology: Theory and applications. Ann. Rev. Nucl. Part. Sci. 60: 27.

Armendáriz-Picón, C., 2007. Creating statistically anisotropic and inhomogeneous perturbations. JCAP 0709: 014.

Axelsson, M., et al., 2013. Directional dependence of $\Lambda \mathrm{CDM}$ cosmological parameters. Astrophys. J. 773: L3.

BICEP2 Collaboration, Ade P. A. R., et al., 2014. BICEP2 I: Detection of B-mode polarization at degree angular scales. arXiv:1403.3985 [astro-ph.CO].

Cai R.-G., Ma Y.-Z., Tang B., Tuo Z.-L. 2013. Constraining the anisotropic expansion of the Universe. Phys. Rev. D 87: 123522 .

Campanelli P., Cea G., Fogli G., Marrone A. 2011. Testing the isotropy of the Universe with type Ia supernovae. Phys. Rev. D 83: 103503.

Dine M. 2007. Supersymmetry and string theory: Beyond the standard model. Cambridge University Press, Cambridge - UK.

Golovnev A., 2010. Linear perturbations in vector inflation and stability issues. Phys. Rev. D 81: 023514.

Golovnev A., 2011. On cosmic inflation in vector field theories. Class. Quant. Grav 28: 245018.

Golovnev A., Mukhanov V., Vanchurin V. 2008.Vector inflation. JCAP 0806: 009.

Greene P. B., Kofman L., Linde A. D., Starobinsky, A. A. 1997. Structure of resonance in preheating after inflation. Phys. Rev. D 56: 6175.

Groeneboom N. E., Ackerman L., Wehus I. K., Eriksen H. K. 2010. Bayesian analysis of an anisotropic universe model: Systematics and polarization, Astrophys. J. 722: 452.

Groeneboom N. E., Eriksen H. K. 2009. Bayesian analysis of sparse anisotropic universe models and application to the 5-yr WMAP data. Astrophys. J. 690: 1807.

Himmetoglu B., Contaldi C. R., Peloso M. 2009a. Instability of the Ackerman-Carroll-Wise model, and problems with massive vectors during inflation. Phys. Rev. D 79: 063517.

Himmetoglu B., Contaldi C. R., Peloso M., 2009b.Instability of anisotropic cosmological solutions supported by vector fields. Phys. Rev. Lett. 102: 111301.

Himmetoglu B., Contaldi C. R., Peloso M. 2009c.Ghost instabilities of cosmological models with vector fields non-minimally coupled to the curvature. Phys. Rev. D 80: 123530.

Kalus B., Schwarz D. J., Seikel M., Wiegand A. 2013. Constraints on anisotropic cosmic expansion from supernovae. Astron. Astrophys. 553: A56.

Kane G. L. 1993. Modern elementary particle physics: The fundamental particles and forces. Addison-Wesley Press, Boston - USA.

Kim J., Komatsu E. 2013. Limits on anisotropic inflation from the Planck data. Phys. Rev. D 88: 101301.
Kim C. W., Murphy P. 1985. On the minimum amount of inflation to solve the flatness and horizon problems. Phys. Rev. D 32: 3303 .

Lyth D. H., Liddle A. R. 2009. The primordial density perturbation: Cosmology, inflation, and the origin of structure. Cambridge University Press, Cambridge - UK.

Maleknejad A., Sheikh-Jabbari M. M. 2011. Non-abelian gauge field inflation. Phys. Rev. D 84: 043515.

Maleknejad A., Sheikh-Jabbari M. M. 2013. Gauge-flation: Inflation from non-abelian gauge fields. Phys. Lett. B 723: 224.

Maleknejad A., Sheikh-Jabbari M. M., Soda J. 2012. Gaugeflation and cosmic no-hair conjecture. JCAP 1201: 016.

Maleknejad A., Sheikh-Jabbari M. M., Soda J. 2012. Gauge fields and inflation. Phys. Rep. 528: 161.

Mukhanov V. 2005. Physical foundations of cosmology. Cambridge University Press, Cambridge - UK.

Paci F., et al. 2013. Hemispherical power asymmetries in the WMAP 7-year low-resolution temperature and polarization maps. arXiv:1301.5195 [astro-ph.CO].

Penzias A. A., Wilson R. W. 1965. A measurement of excess antenna temperature at $4080-M c / s$, Astrophys. J. 142: 419.

Planck Collaboration, Ade, et al., 2013a. Planck 2013 results. XVI. Cosmological parameters. arXiv:1303.5076 [astro-ph.CO].

Planck Collaboration, Ade, et al., 2013b. Planck 2013 results. XXIII. Isotropy and statistics from the CMB. arXiv:1303.5083 [astro-ph.CO].

Ramazanov S. R., Rubtsov G. 2014. Constraining anisotropic models of the early Universe with WMAP9 data. Phys. Rev. D 89: 043517.

Rubakov V. A. 1982. Graviton creation in the inflationary universe and the grand unification scale. Phys. Lett. 115B: 189.

Ryder L. H. 1985. Quantum field theory. Cambridge University Press, Cambridge - UK.

Singal A. K. 2013. Is there a violation of the Copernican principle in radio sky? arXiv:1305.4134 [astro-ph.CO].

Starobinsky A. A. 1980. A new type of isotropic cosmological models without singularity. Phys. Lett. 91B: 99.

Starobinsky A. A. 1982. Dynamics of phase transition in the new inflationary universe scenario and generation of perturbations. Phys. Lett. 117B: 175.

Weinberg S. 1996. The quantum theory of fields, Volume 2: Modern applications. Cambridge University Press, Cambridge - UK.

Weinberg S. 2008. Cosmology. Oxford University Press, Oxford - UK.

WMAP Collaboration, Hinshaw, G., et al., 2013. Nine-year Wilkinson Microwave Anisotropy Probe (WMAP) observations: Cosmological parameter results. Astrophys. J. Suppl. Ser. 208: 19.

Zhao W., Wu P., Zhang Y. 2013. Anisotropy of cosmic acceleration. Int. J. Mod. Phys. D 22: 1350060. 